\title{
The European programme BORIS (Bioavailability Of Radionuclides In Soils): A global analysis of results
}

\author{
C. Tamponnet ${ }^{1, *}$, A. Martin-Garin ${ }^{1}$, M.-A. Gonze ${ }^{1}$, N. Parekh ${ }^{2}$, R. Vallejo ${ }^{3}$, \\ T. Sauras ${ }^{3}$, J. Casadesus ${ }^{3}$, C. Plassard ${ }^{4}$, S. Staunton ${ }^{4}$, M. Norden ${ }^{5}$, \\ R. Avila ${ }^{5}$, G. Shaw ${ }^{6}$ and C. Wells ${ }^{6}$ \\ ${ }^{1}$ Institute of Radioprotection and Nuclear Safety, DEI/SECRE, CADARACHE, BP. 1, \\ 13108 Saint-Paul-lez-Durance Cedex, France, e-mail: christian.tamponnet@irsn.fr \\ ${ }^{2}$ Center for Ecology and Hydrology, Lancaster Environment Centre, Lancaster University, \\ Lancaster LA1 4YQ, UK \\ ${ }^{3}$ Department of Plant Biology, University of Barcelona, 08028 Barcelona, Spain \\ ${ }^{4}$ INRA, Soil Science Unit, 34060 Montpellier, France \\ ${ }^{5}$ Swedish Radiation Protection Institute, 17116 Stockholm, Sweden \\ ${ }^{6}$ Imperial College at Silwood Park, Ascot, SL5 5PY Berkshire, UK \\ * To whom all correspondence should be addressed
}

\begin{abstract}
The ability to predict the consequences of an accidental release of radionuclides relies mainly on the level of understanding of the mechanisms involved in radionuclides interactions with different components of agricultural and natural ecosystems and their formalisation into predictive models. Numerous studies and databases about contaminated agricultural and natural areas have been obtained but their use to enhance our prediction ability has been largely limited by their unresolved variability. Such variability seems to stem from incomplete knowledge about radionuclide interactions with the soil matrix, soil moisture, biological elements in the soil and additional pollutants, which may be found in such soils.

In this project, we investigated mainly the role of the biological elements (plants, mycorrhiza, microbes) in: radionuclide sorption/desorption in soils and radionuclide uptake/release by plants. Because of their chemical nature importance, the bioavailability of three radionuclides: caesium, strontium, and technetium have been followed. The role of one additional non-radioactive pollutant (copper) has been scrutinised.

Role of microorganisms ( $\mathrm{K}_{\mathrm{d}}$ for caesium and strontium in organic soils is much greater in the presence of microorganisms than in their absence), plant physiology (changes in plant physiology affect radionuclide uptake by plants), the presence of mycorrhizal fungi (interferes with the uptake of radionuclides by plants), have been demonstrated.

Knowledge acquired from these experiments has been incorporated into two mechanistic models CHEMFAST and BIORUR specifically modelling radionuclide sorption/desorption from soil matrices and radionuclide uptake by/release from plants. These mechanistic models have been incorporated into an assessment model to enhance its prediction ability.
\end{abstract}

\section{INTRODUCTION}

Numerous studies and databases about contaminated agricultural and natural areas have been obtained from previous contamination events including nuclear accidents. The use of these previous data to enhance our prediction ability has been largely limited by their unresolved variability. Such variability seems to proceed from a lack of knowledge about radionuclide interactions with the soil matrix, soil moisture, biological elements in the soil and additional pollutants, which may be found in such soils [1]. To improve our understanding of the mechanisms governing the transfer of radionuclides to plants, we 
apprehended the role of abiotic components: soil elements and structure and of biotic components: soil microorganisms, plants, mycorrhiza, and the association of these three biological components. To improve existing predictive models of radionuclide interaction with soils by incorporating the knowledge acquired from the experimental results, we use a three-step procedure: (1) specifying a conceptual and compartment model based initially on the existing knowledge and on previously developed models; (2) developing mechanistic models of the processes occurring between the soil solid phase (mineral, organic, microbial) and the soil solution, and of the processes occurring between the plant system (including eventual mycorrhiza) and the soil solution, and validating these mechanistic models with the experimental results; and (3) integrating the results obtained into an improved compartment model with reduced uncertainties of predictions compared to those of previous models.

Because of the size limit of this paper, only major results will be presented.

\section{ROLE OF ABIOTIC COMPONENTS}

For the sake of homogeneity defined as a major factor of experimental works by the modellers, a considerable effort was put into identifying, locating and sampling a suitable agricultural soil for use in both the full scale column experiments and in the associated laboratory experiments. After a global consultation, a silty loam soil (Batcombe series) from southern England was selected.

\subsection{Specific role of soil components on the radionuclide bioavailability}

We have studied the adsorption and desorption of $\mathrm{Cs}$ and $\mathrm{Sr}$ on synthetic soils consisting of individual solid reference materials, or simple binary or ternary mixtures of model soil components. Because of the very small adsorption of Tc on soils and soil components, it was not included in this study.

From these experiments, the adsorption properties of $\mathrm{Sr}$ on mineral soil components are simple, and not influenced by inorganic or organic coatings. Reference clay minerals are therefore good models to predict and explain the mobility of $\mathrm{Sr}$ in soil systems. In contrast, the adsorption of $\mathrm{Cs}$ depends in a complex manner on inorganic and inorganic coatings. Care must be taken in extrapolating adsorption data obtained using reference minerals to soils.

\subsection{Role of soil structure on radionuclide bioavailability}

$\mathrm{Cs}, \mathrm{Tc}$ and $\mathrm{Sr}$ incubation experiments were performed in different physico-chemical conditions. Its shows the strong affinity of Cs for the solid phase, the low affinity of Tc and its dependence upon the presence of microorganisms, and the quasi-absence of effects of changing conditions on $\mathrm{Sr}$ affinity for soil matrix.

\section{ROLE OF BIOTIC COMPONENTS}

\subsection{Role of soil microorganisms in radionuclide bioavailability in soils}

A mineral free organic matter was produced using deciduous leaves and was stored at $-20^{\circ} \mathrm{C}$ in $500 \mathrm{~g}$ aliquots. For direct comparison of biotic and abiotic components, two sets of autoclaved material are used. One sample is re-inoculated with a soil extract solution (in sterile distilled water - SDW) and the other is amended with an equal quantity of SDW. 
To check the role of microbes on radionuclide behaviour [2], the effect of added clay minerals (montmorillonite and illite) in litter on the uptake of Sr-85 and Cs-137 was studied. The distribution ratio, $\mathrm{Kd}$, for ${ }^{85} \mathrm{Sr}$ and ${ }^{137} \mathrm{Cs}$ in abiotic versus biotic microcosms is shown in Figure 1.
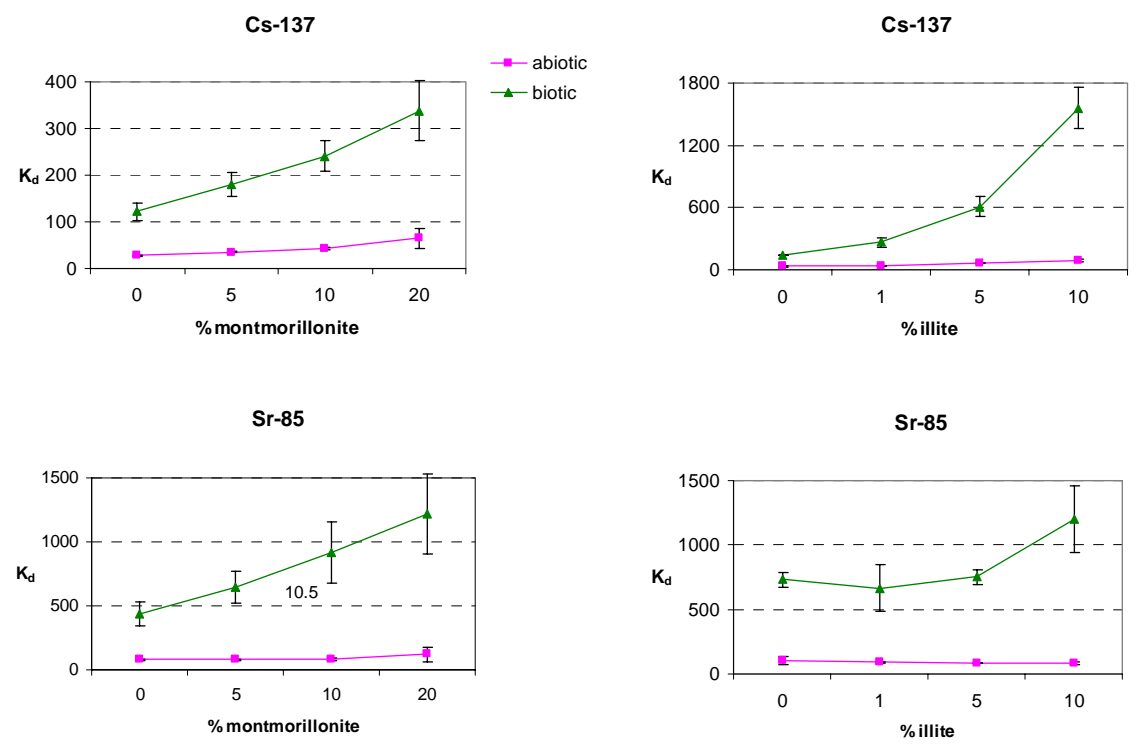

Figure 1. Calculated distribution ratio for ${ }^{137} \mathrm{Cs}$ and ${ }^{85} \mathrm{Sr}$ in biotic and abiotic microcosms following 14 days incubation, for different amendments of montmorillonite and illite to an organic matrix.

The following observations were noted from the results:

$\checkmark . \mathrm{K}_{\mathrm{d}}$ biotic $>\mathrm{K}_{\mathrm{d}}$ abiotic suggesting the possible role of microbes in the sorption of both radionuclides $\checkmark$. High degree of variability observed in the biotic $\mathrm{K}_{\mathrm{d}}$ values

$\checkmark . \mathrm{K}_{\mathrm{d}}$ for ${ }^{137} \mathrm{Cs}$ increased with increasing amounts of clay, this was more pronounced in the biotic system. $\checkmark . \mathrm{K}_{\mathrm{d}}$ for ${ }^{137} \mathrm{Cs}$ was higher in the presence of illite than montmorillonite

$\checkmark . \mathrm{K}_{\mathrm{d}}$ for ${ }^{85} \mathrm{Sr}$ was similar in presence of both types of clay

\subsection{Identifying Plant physiological mechanisms involved in radionuclide transfer to plants}

Soil to plant transfer is not determined only by the concentration of radionuclides in the soil solution [3]. Therefore, a more mechanistic approach should replace the use of empirically obtained transfer factors. It has been established [4] that the $\mathrm{K}^{+}, \mathrm{Ca}^{2+}$ and $\mathrm{NO}_{3}{ }^{-}$concentrations in the root medium largely affect the root uptake of $\mathrm{Cs}^{+}, \mathrm{Sr}^{2+}$ and $\mathrm{TcO}_{4}^{-}$respectively.

The effect of plant. K uptake was significantly higher in young plants (Figure 2) with respect to old plants. In addition some effect of $\mathrm{K}$ starvation appeared after 24 hours in contact with the $0.2 \mathrm{mM} \mathrm{K}$ supply medium. Old plants, with low demand presented lower Cs uptake stimulation though there was still a clear effect of K-starvation on Cs root uptake in agreement with results found in the previous experiment. In plants with high demand (young plants), the higher Cs uptake under $0.2 \mathrm{mM} \mathrm{K}$ treatment highlighted the low discrimination between $\mathrm{Cs}$ and $\mathrm{K}$ when the high-K affinity transport system is dominating.

It has been described elsewhere that plant regulation of Cs uptake is mediated by the expression of high affinity $\mathrm{K}$ transport system and the transition between carrier and channel-mediated $\mathrm{K}$ transport systems. For $\mathrm{Ca}$ and $\mathrm{Sr}$, the transport regulation is not as well documented as for $\mathrm{K}$ although some carrier mediated transport for $\mathrm{Ca}$ in conditions of low $\mathrm{Ca}$ in the medium has been described. For $\mathrm{Sr}$ the 
main pathway of uptake is through mass flow. In our experiments we have forced the plant to change the transport systems, hence producing contrasted $\mathrm{Cs}$ and $\mathrm{Sr}$ uptake patterns. According to our results, the uptake of caesium and strontium have been modulated by the plant to keep a target flux of either $\mathrm{K}$ or $\mathrm{Ca}$. Therefore we confirm the hypothesis of plant as a key regulator of soil-to-plant transfer of Cs and $\mathrm{Sr}$.
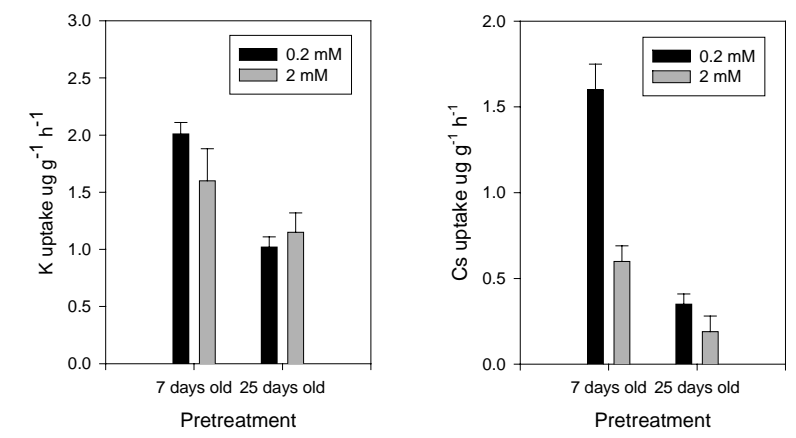

Figure 2. $\mathrm{K}$ and $\mathrm{Cs}$ root uptake at two different plant ages ( 7 and 25 days old plants) after 24 hour growing in two contrasted K supply ( 0.2 and $2 \mathrm{mM} \mathrm{K})$ solution culture.

\subsection{Role of mycorrhizal fungi in radionuclide bioavailability in soils}

The aim of this experimental work is to determine how ectomycorrhizal infection can modify the uptake and translocation of radionuclides [5]. One set of experiments is presented in this paper dealing with the effect of ectomycorrhizal symbiosis on the radionuclide uptake and the role of cupper (additional pollutant) on that uptake.

Rhizopogon roseolus was grown in association with Pinus pinaster. At the time of harvest (after 4 months of growth in rhizotrons), macroscopic examination of fungal development indicated that the colonization of plant compartment by $R$. roseolus hyphae was good. The results (Fig. 3) showed that mycorrhizal effects on radionuclides in plants varied with the radionuclide type and the $\mathrm{Cu}$ level. Indeed, the presence of the ectomycorrhizal fungus decreased the accumulation of ${ }^{95 \mathrm{~m}} \mathrm{Tc}$ in the shoots of mycorrhizal plants, did not modify ${ }^{85} \mathrm{Sr}$ accumulation in roots or shoots of mycorrhizal plants and increased greatly ${ }^{137} \mathrm{Cs}$ accumulation in roots and shoots of mycorrhizal plants when the soil did not contain any copper.
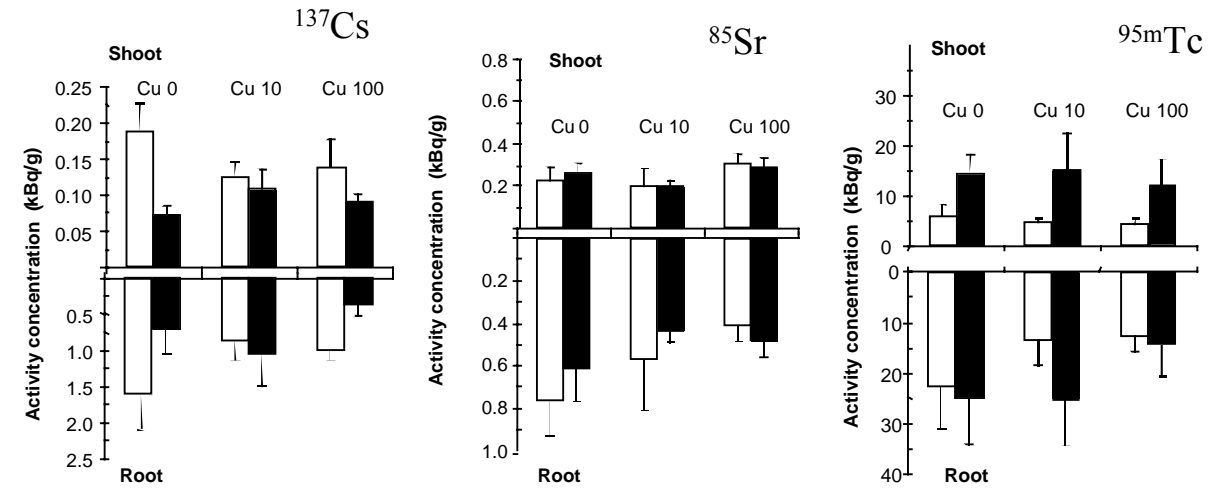

Figure 3. Activity concentration $(\mathrm{kBq} / \mathrm{g}$ fw) shoots and roots of Pinus pinaster plants, whether associated with the ectomycorrhizal fungus (white boxes) Rhizopogon roseolus or non mycorrhizal (black boxes). Two-month old plants were grown for 4 months in rhizotrons containing a thin layer of Batcombe soil not amended (Soil) or amended with ${ }^{95 \mathrm{~m}} \mathrm{Tc},{ }^{85} \mathrm{Sr}$ and ${ }^{137} \mathrm{Cs}(\mathrm{RN})$ or radionuclides and copper at $10 \mathrm{mg} \mathrm{kg}^{-1}$ (RN, Cu 10) or $100 \mathrm{mg} \mathrm{kg}^{-1}$ of soil ( $\mathrm{RN}, \mathrm{Cu} 100)$. Data are means \pm standard deviation $(\mathrm{n}=6)$. 


\subsection{Global role of biological components in radionuclide bioavailability in soils}

Such role is scrutinized by implementing soil column experiments to determine in situ $\mathrm{K}_{\mathrm{d}}$ values for $\mathrm{Cs}$, Sr and Tc in Batcombe soil. After contamination with ${ }^{137} \mathrm{Cs},{ }^{85} \mathrm{Sr}$ and ${ }^{95 \mathrm{~m}} \mathrm{Tc}$ the selected soils were packed to a constant bulk density of $1.1 \mathrm{~g} \mathrm{~cm}^{-3}$ into columns of $50 \mathrm{~cm}$ height and $15 \mathrm{~cm}$ diameter. Sterilisation of half the columns was achieved by fumigation with $\mathrm{CH}_{3} \mathrm{Br}$ and the column was either planted with the experimental plant species of interest (wheat), or left unplanted.

Soil solution samples were collected and analysed for radionuclide activity concentrations and stable element chemistry (cations and anions). TDR and redox measurements were taken at approximately fortnightly intervals using in situ probes. In situ gamma measurements were taken at two monthly intervals.

The results of primary importance are the in situ $\mathrm{K}_{\mathrm{d}}$ values for ${ }^{137} \mathrm{Cs},{ }^{85} \mathrm{Sr}$ and ${ }^{95 \mathrm{~m}} \mathrm{Tc}$. Ranges of $\mathrm{K}_{\mathrm{d}}$ values for one of the eight experimental columns were $0.69-171$ for ${ }^{95 \mathrm{~m}} \mathrm{Tc}, 1.96-238$ for ${ }^{85} \mathrm{Sr}$ and $39.7-9431$ for ${ }^{137} \mathrm{Cs}$. These in situ $\mathrm{K}_{\mathrm{d}}$ values are within the expected ranges for each of these radionuclides. However, the variability within these ranges was completely systematic with a monotonic increase in $\mathrm{K}_{\mathrm{d}}$ from lowest to highest values from the dry surface of the column to the saturated region below the water table at $45-50 \mathrm{~cm}$ depth. This reflects the observation that soil solution ion concentrations of both radionuclides and stable elements declined monotonically from surface to the saturated deeper part of the soil columns.

Other factors, which showed a systematic variation with soil depth, were soil moisture content (increased from top to bottom), soil redox potential (decreased from top to bottom) and soil $\mathrm{pH}$ (increased from top to bottom). Each of the eight columns has yielded 40 to 50 estimates of in situ $\mathrm{K}_{\mathrm{d}}$ for each radionuclide and this, together with the supporting data concerning stable element concentrations and soil physico-chemical parameters, provides a substantial database, which will be used to determine the key parameters controlling in situ $\mathrm{K}_{\mathrm{d}}$ for $\mathrm{Cs}$, $\mathrm{Sr}$ and Tc.

In conclusion, solution concentrations of major ions and radionuclides show a decrease from maximum values in the dry soil at the column surfaces to the saturated lower column regions. This is mirrored by a monotonic increase in in situ $\mathrm{K}_{\mathrm{d}}$ values of radionuclides from minima in dry surface soil to saturated soil at and below the water table.

\section{IMPROVING PREDICITIVE MODELS}

The usual approach for evaluating radioactive nuclide uptake by plants from soil is by using concentration ratios, $\mathrm{CR}$, which is defined as the ratio of radionuclide concentration in plants to that in soil at a specified depth. This approach is recommended by the IAEA to use in modelling radionuclide uptake. A review shows that the caesium CR for agricultural plants can vary by a factor of up to 25 even for plants grown on the same site. Different types of soils lead to a variation by a factor of 50 . A variation from two to three orders of magnitude of the CR from soil to forest plants has been reported. In this project we explore a possible solution to this problem by describing the bioavailability of radioactive nuclides in soils in mechanistic terms [6,7].

\subsection{Soil/soil solution partition of radionuclides CHEMFAST biogeochemical model}

The CHEMFAST model is a Column Heuristic Model of radionuclide Fixation and Solution Transport. Heuristic models are based on progressive learning of system behaviour - in the BORIS programme learning is based on experiments. The CHEMFAST model addresses vertical transport of radionuclides in the solution phase by solving the advection dispersion using an implicit finite difference technique. This has been found to reproduce the migration behaviour of non-sorbed radioactive solutes such as ${ }^{36} \mathrm{Cl}^{-}$and ${ }^{3} \mathrm{HHO}$. Sorption and desorption between the solid and liquid phases are represented as kinetic processes which are first order with respect to time but which vary in a non-linear manner with respect to solution concentration. 
Concentration-dependent sorption-desorption of radionuclides is based on the Langmuir formulation. The rate of sorption to a surface should be proportional to a driving force times the area of the surface sites available for sorption. The effective driving force is the concentration in the liquid phase.

\subsection{Soil solution/plant transfer of radionuclides BIORUR model}

The main concept is that radionuclides move in plants and ecosystems through the same pathways as other elements and the activities of these pathways vary between scenarios in a way which can be explained (and predicted) by known rules of nutrient cycling. In this sense, the algorithms of BIORUR do not pretend to describe in mechanistic terms all the involved process because this would be extremely complex. Instead, the algorithms of BIORUR focus at the difference between the behaviour of a radionuclide and that of its nutrient analogue in the most relevant pathways, in order to translate the flux of nutrients in a given scenario to the corresponding flux of radionuclides.

BIORUR considers the soil composed by a number of soil layers, which in turn are composed by three compartments: 1) soil solution and mineral soil, 2) organic mater and 3) roots. The pathways between these compartments are root uptake, mineralization of organic mater and mycorrhizal transfer. Similarly, plants are composed by four compartments, 1) roots, 2) functional leaves, 3) growing parts and 4) storage pool, and the pathways between plant compartments are either xylem flow, or phloem flow. The algorithms of BIORUR describe the flux of radionuclides between compartments in terms of flux of nutrients and a selectivity coefficient for radionuclide compared to nutrient, where the flux of nutrient is an input parameter, which characterize the scenario, while the selectivity coefficient is assumed a constant property of the pathway, valid for any scenario.

\subsection{Development and validation of a predictive model}

A literature review on the use of the concept of bioavailability in studies of the soil-to-plant transfer of radionuclides and other contaminants, as well as in studies of nutrients cycling in the soil-plant system, was carried out. Alternative operational definitions of bioavailability were proposed and how to use this concept in the assessment model. This issue was considered in detail and a complete strategy and plan for the integration of the mechanistic model and the experimental data in the assessment model was formulated. Alternative formulations of the assessment model were developed at the conceptual and mathematical level, i.e. without including parameterisation.

\section{Acknowledgments}

This research programme has been funded by the $5^{\text {th }}$ European Framework Programme/ EURATOM.

\section{References}

[1] Tamponnet C. et al., Radioprotection, 37 (2002) 331-336.

[2] Tamponnet C. et al., "Impact of micro-organisms on the fate of radionuclides in rhizospheric soils", Radioactive pollutants - Impact on the environment, F. Bréchignac and B. Howard Eds, (EDP Sciences, Les Ulis, 2001) pp. 175-185.

[3] Shaw G. and Bell J.N.B., J. Environ. Radioact., 13 (1991) 283-296.

[4] Sauras Yera T. et al., J. Environ. Radioact., 45 (1999) 191-217.

[5] Entry J. A. et al., Environ. Pollution 104(3) 449-457

[6] Avila R. and Moberg L., J. Environ. Radioact., 45 (1999) 271-282.

[7] Avila R. et al., BORIS Technical Note 1: Model specifications (IRSN/DPRE/SERLAB Report 2001-028, Cadarache, 2001). 